\title{
Automated convolutional neural network approach for discriminating systemic sclerosis on the basis of hand thermal pattern
}

\author{
by C. Filippini ${ }^{\star}$, D. Cardone*, A.M. Chiarelli* ${ }^{*}$ D. Perpetuini ${ }^{\star}$, P. Amerio** A. Merla* \\ * Department of Neurosciences, Imaging and Clinical Sciences, University G. d'Annunzio of Chieti-Pescara, Italy \\ ** Department of Medicine and Aging Science, Dermatologic Clinic, G. D’Annunzio University, Chieti, Italy
}

\begin{abstract}
Systemic sclerosis (SSc) is a disorder of microvessels and connective tissue, characterized by fibrosis and vascular obliteration. Limbs extremities, including hands, are majorly involved, and they present inhomogeneous patterns of injury. The diagnosis of the disease can be performed using infrared (IR) imaging. Standard procedure includes the recording of IR images of the hands before and after exposure to cold stress, and evaluation of temperature recovery in manually selected regions of interest. A completely automated methodology based on convolutional neural network is here introduced with the purpose of classifying SSc relying uniquely on resting condition images of the hands.
\end{abstract}

\section{Introduction}

Systemic sclerosis (SSc) is a heterogeneous connective tissue disease characterized by small vessel vasculopathy, production of autoantibodies, and fibroblast dysfunction leading to increased deposition of extracellular matrix [1]. SSc is approximately eight times more common among women than men [2]. It is most common in the third to fifth decades of life and is rare in children [3,4]. Clinical symptoms of the disease include peripheral and persistent microvascular vasoconstriction, swelling of the fingers, skin tightening and contractures of the fingers, polyarthralgia, and possibly dysphagia [3,5]. The most common initial symptoms and signs are vascular, such as Raynaud phenomenon, which is often long-lasting, as well as insidious swelling of the distal extremities (i.e. swollen fingers), followed by gradual thickening of the skin of the face and fingers with late skin ulcers $[3,6,7]$. SSc results in skin, tendon, joint, and vessel damage leading to worsening of life's quality [8,9], due to problems of functioning and restricted participation in society [10]. Hands involvement is often the first clinical manifestation in SSc [11]. It occurs mainly due to skin and periarticular thickening causing significant contracture and limitation of finger movements and hand deformity. Specific treatment of SSc is difficult, and remains a great challenges to the clinician [12], at the moment, no approved treatment is available that completely interrupts the natural progression of the 'clinically recognizable' disease [3]. Consequently, efforts are being made to study the disease in its early stages, before overt disease and irreversible organ damage have occurred. Several tools are currently available to assess the disease status, including devices to evaluate skin thickness (i.e., plicometer [13]) and blood flow perfusion (i.e., laser Doppler flowmetry [14]; ultrasound [15]). Thermal infrared (IR) imaging is also employed to evaluate the thermoregulatory finger properties and identify SSc [16].

Thermal IR imaging is a well-known and widely used imaging technique that enables contactless assessment of the skin thermal distribution [17-19]. Capturing the infrared radiation spontaneously emitted by the body, the thermal camera is able to convert it into a digital map of the body superficial temperature profile $[20,21]$. The technique has become an important tool in medical research, thanks to its contactless, non-invasive, and security features [22,23]. IR imaging studies for SSc evaluation are usually performed by monitoring fingers responses to a controlled cold stress [24-26]. IR images of the dorsum of the subject's hands are recorded before and after a cold stimulus. The first ones are necessary to obtain the baseline of the fingers' temperature and the remaining ones are useful to monitor the temperature recovery. Usually, subjects seat in an environment-controlled room (stable temperature around $23^{\circ} \mathrm{C}$ and humidity around $50-60 \%$ ) with both hands place on a table covered with a nonreflective black sheet [16]. Before undergoing measurements, the subjects observe an acclimatization period of $20 \mathrm{~min}$ in the recording room. The measurement commonly lasts 25 min including both baseline and recovery phases. The cold stress is achieved by immersing the hands for 2 min in a $3 L$ water bath maintained at $10^{\circ} \mathrm{C}$ [25]. After the cold stress, the hands should be placed in the same position as before the cold stress. Importantly, baseline temperature and its recovery to the stress are evaluated on manually selected regions of interest (ROls) located on the fingers. The manual identification of ROls may indeed reduce the information content encoded in the original images as well as being affected by human error.

In order to avoid biases associated with the manual selection of the ROIs and the related features, automated classification procedures are preferable. Among these procedures, convolutional neural network (CNN) is a class of deep neural networks, most commonly applied to analyse visual imagery [27], that allow to classify different class without apriori assumptions. In recent years, CNNs have achieved great success in many computer vision tasks [28]. Deep learning allows computational models that are composed of multiple processing layers to learn representations of data with multiple levels of abstraction [29]. The multi-layered neural network mimics the human neural circuit structure. Indeed, CNNs draw biological inspiration from the visual cortex [30]. The visual cortex has small regions of cells that are sensitive to specific regions of the visual field. In detail, some individual neuronal cells in the brain responded (or fired) only in the presence of edges of a certain orientation $[31,32]$. For example, some neurons fired when exposed to horizontal edges and other when 
vertical or diagonal edges are shown. This idea of specialized components within a system that has specific tasks (neuronal cells in the visual cortex in search of specific characteristics) is the basis behind CNNs [33]. Thanks to their structure, CNNs can automatically extract features from an image, unlike classical classification methods that still require feature extraction by a human expert [34]. Since the great performances of this classification method, CNNs have been applied also in the biomedical signal processing. For instance, they have been used for cardiovascular aging assessment through photoplethysmographic signal [35], for biomedical imaging segmentation [36], myocardial infraction diagnosis [37] and affective state recognition trough thermal IR imaging [18].

In this study, a novel automated classification methodology that allows to distinguish SSc patients from healthy controls is presented. This new method relies on CNNs applied to resting condition thermal images of the hands. The procedure adopted allows robust identification of SSc through completely automated analysis of the temperature pattern of the whole hands. It is worth noticing that the proposed procedure does not require the administration of a cold stress stimulus, providing a quick method for SSc diagnosis suitable for large scale clinical applications.

\section{Materials and Methods}

\subsection{Participants}

51 subjects participated in this study, 26 healthy and 25 SSc subjects. The study was authorized by the Human Board Review and Local Ethical Committee of the School of Medicine of the University of Chieti-Pescara. The measurements were performed in agreement with the ethical standards of the Helsinki Declaration [38]. All subjects gave written informed consent before participating in the study. Participation was strictly voluntary.

\subsection{Experimental paradigm}

Prior to testing, each subject was left in the experimental room for about 10-15 minutes in order to allow participants to achieve proper acclimatization to the room environmental conditions and the baseline skin temperature to stabilize [39]. The recording room was set at a standardized temperature $\left(23^{\circ} \mathrm{C}\right)$ and humidity $(50-60 \%)$ by a thermostat according to the International Academy of Thermology (IACT) guidelines [40]. Participants sat comfortably on a chair during acclimatization and measurement periods. The experimental measure consisted in the acquisition of a single thermal image of both subject's hands in a resting condition. The subject was asked to place his/her hands on a sheet in which the hands' shape were drawn, in order to establish and maintain the same position for all subjects.

\subsection{Thermal IR imaging acquisition}

IR images of the hands were recorded by means of a digital thermal camera (FLIR SC3000, FlirSystems, Sweden), with a Focal Plane Array of $320 \times 240$ Quantum Well Infrared Photodetector (QWIP) detectors, capable of collecting the thermal radiation in the 8-9 $\mu \mathrm{m}$ band, with a $0.02-\mathrm{s}$ time resolution, and $0.02 \mathrm{~K}$ temperature sensitivity/noise equivalent temperature difference (NETD). The thermal camera response was blackbody-calibrated to null noise-effects related to the sensor drift/shift dynamics and optical artefacts. Cutaneous emissivity was estimated as $\varepsilon \approx 0.98$. This value was widely approved and adopted in the literature [41-45].

\subsection{Data analysis}

Firstly, the IR images were downsampled from $320 \times 240$ to $80 \times 107$ pixels and normalized by subtracting the average value of the entire set of images. A CNN was, then, used to classify the images into two classes: healthy and SSc subjects. The structure of the CNN employed in this work was composed of 3 convolutional layers and one fully connected layer prior to the output neuron. The input layer was composed of the down-sampled normalized thermal IR image of the subject's hands. The convolutional layers consisted of convolutional (filtering), non-linear and pooling layers which performed features extraction and dimension reduction [46]. For each convolutional layer, 10 filters were employed. The filters' size was $5 \times 5$ with a stride of 1 [35]. As a non-linear processing function, the Rectified Linear Unit (ReLU) function was employed. In fact, the ReLu function was proven to dampen the vanishing gradient problem, providing better performance than other non-linear functions (such as the hyperbolic tangent or the sigmoid function) [47]. For the pooling layer, MaxPooling was utilized, in which the largest element from the rectified feature map is retained [48]. A $5 \times 5$ MaxPooling was implemented to reduce the dimensionality of each feature to 1 after the three convolutional layers and before the fully connected layer. The fully connected layer was composed of 20 neurons. Lastly, a softmax function was 
applied, which takes as input a vector of real numbers and normalizes it into a probability distribution consisting of probabilities proportional to the exponentials of the input numbers. It has the following form [49]:

$$
y(z)_{i}=\frac{e^{z_{i}}}{\sum_{j=1}^{K} e^{z_{j}}} \quad \text { for } \mathrm{i}=1, \ldots, \mathrm{K} \text { and } \mathrm{z}=\left(\mathrm{z}_{1}, \ldots, \mathrm{ZK}\right)
$$

where $z$ is the input vector and $y$ is the function output. Since the classifier had to discriminate between two classes (Healthy and SSc patients) the output layer was composed of two neurons performing the softamx transformation.

The weights were initialized in a pseudo-random approach employing a truncated normal distribution (mean $=0$, standard deviation $=0.1$, truncation at 2 standard deviations), and the biases have been initialized to a small number approximately equal to 0 [50]. The CNN was trained in a supervised learning manner [51] by employing the cross-entropy as objective function and the Adam Optimizer as optimization algorithm [52]. The learning rate parameter of Adam Optimizer algorithm was set to $10^{-3}$ [52]. The optimization procedure was iterated for 30 epochs with a batch size of 4 . In order to address the CNN generalization performance, we decided to perform a 51-fold cross-validation procedure [53]. The described CNN architecture, training and validation have been implemented in Python through the open-source software library Tensorfow [54]. Further analysis was performed in Matlab. Figure 1a-c shows an example of the input images respectively for healthy and SSc subjects, and Figure 1b-d the feature maps resulting as output from each convolutional layer related to a healthy control and SSc subject.

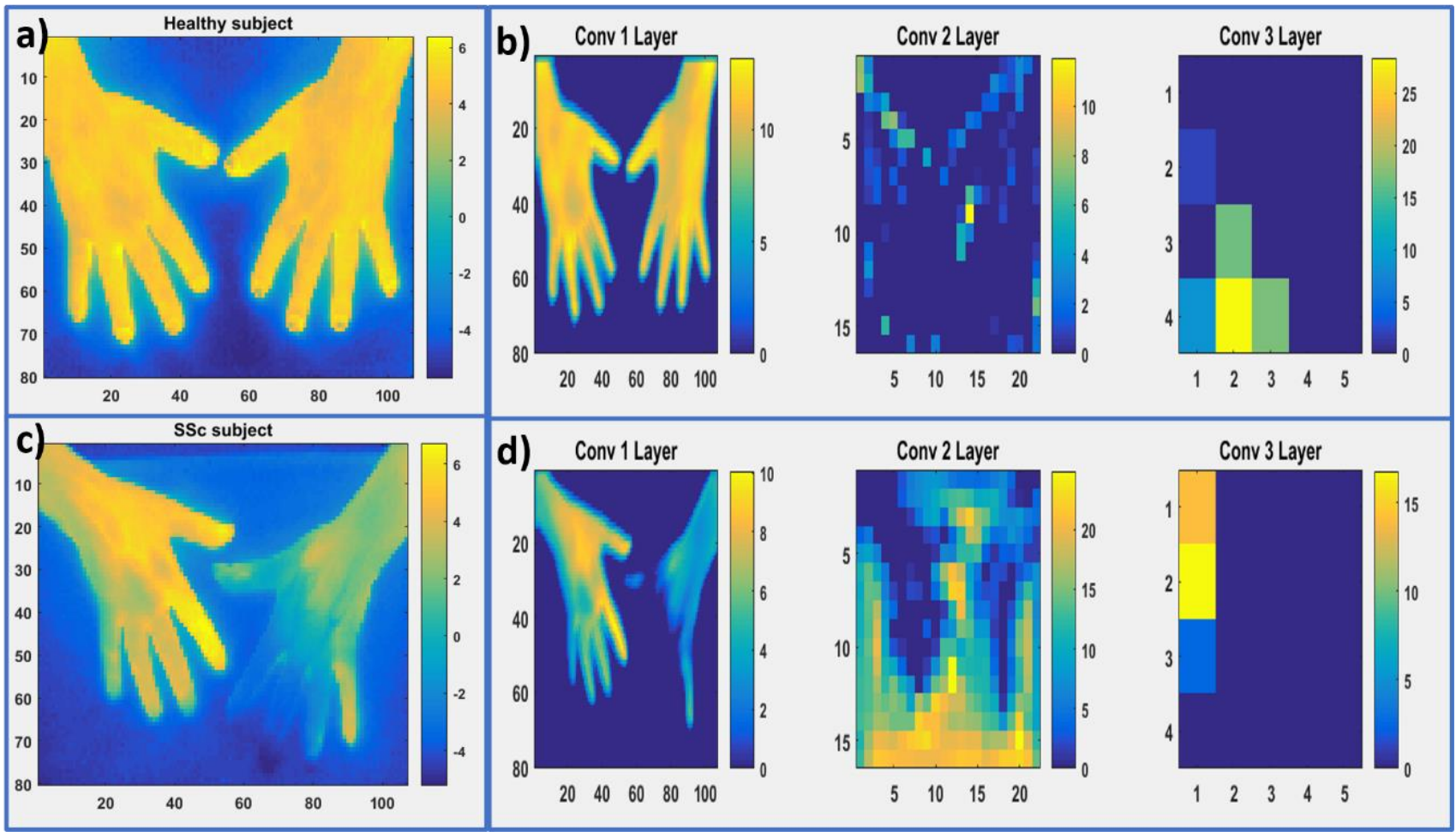

Fig. 1. CNN feature maps. a) Input image of a representative healthy subject within the dataset. b) Features map related to the healthy input images resulting from each convolutional layer. c) Input image of a representative SSc subject within the dataset. d) Features map related to the SSc input images resulting from each convolutional layer.

\section{Results}

The performance of the described procedure is reported in Figure 2 which shows the average accuracy and related standard errors of the CNN employed on the training and testing set as a function of training iterations. The CNN accuracy in test sample reached a plateau value of $82.35 \% \pm 4.9 \%$ and a maximum value of $92.16 \% \pm 4 \%$. The confusion matrix, which allows the visualization of the CNN performance on the classification task is shown in Table 1 and it was performed by counting the number of correct CNN predictors after an argmax evaluation of the CNN output vector, averaged among the plateau iterations. This procedure was conducted following the CNN 51-fold cross validation. 


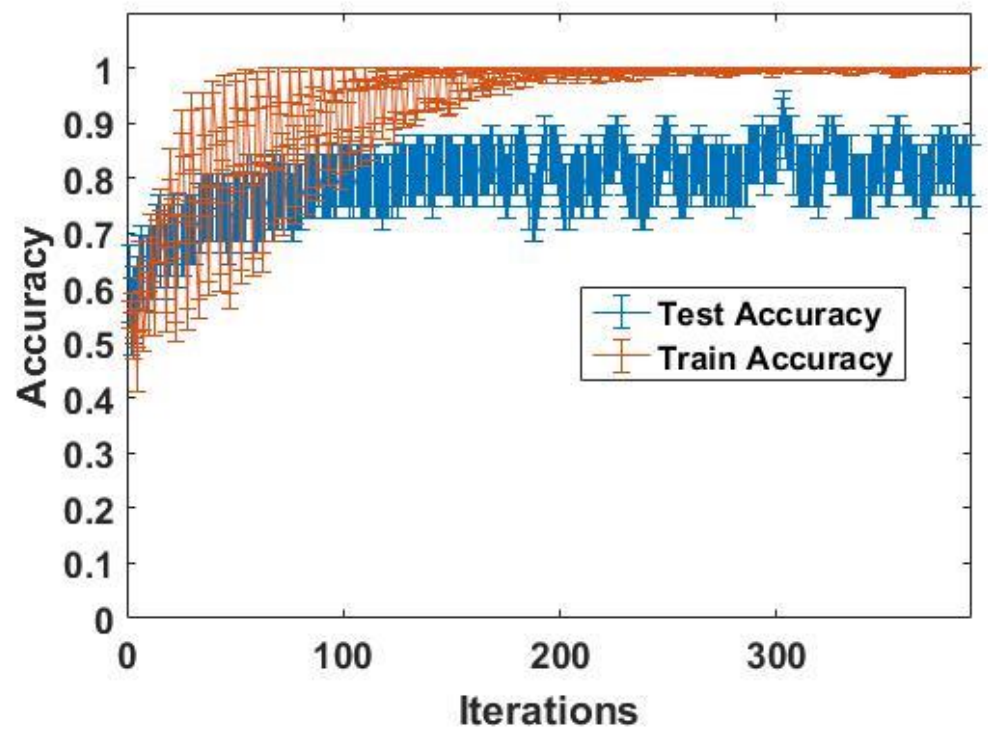

Fig. 2 Average accuracies and related standard errors of the CNN employed on training and test dataset. The accuracy is reported as a function of training iterations.

An overall CNN accuracy of $82.35 \%$ was reached, while a precision level of $80.77 \%$ and $84.00 \%$ was ensured for healthy and SSc subjects, respectively, as shown in the confusion matrix.

Table 1. Confusion matrix showing the overall CNN performance on healthy and SSc subjects classification.

\begin{tabular}{|c|c|c|c|c|}
\hline & & \multicolumn{2}{|c|}{ Actual class } & \\
\hline & & Healthy & SSc & \\
\hline \multirow{3}{*}{ Predicted class } & Healthy & 21 & 4 & \multirow{3}{*}{$\begin{array}{r}\text { Accuracy } \\
82.35 \%\end{array}$} \\
\hline & SSc & 5 & 21 & \\
\hline & Precision & $80.77 \%$ & $84.00 \%$ & \\
\hline
\end{tabular}

\section{Discussion}

$\mathrm{SSc}$ is a heterogeneous connective tissue disease for which, to date, there is no specific treatment that completely interrupts the natural progression of the disease. Consequently, efforts are being made to study the disease in its early stages before it sets in and induces irreversible organ damage. Thermal IR imaging is a currently available technique for assessing the presence of the disease. This technology is employed to evaluate the thermoregulatory finger properties and identify SSc. However, thermal IR imaging studies are usually performed by monitoring the finger's thermal response to cold stress. This procedure lasted about 25 minutes and consisted of immersing the hands in cold water at a temperature of $10^{\circ} \mathrm{C}$. Furthermore, the baseline temperature and its stress recovery were evaluated on manually selected regions of interest located on the fingers. Nonetheless, manual identification of ROls can be influenced by human errors as well as resulting in a reduction of the content encoded in the original images. The procedure introduced in this study allowed a solid and rapid identification of SSc. The classification was performed through a completely automated analysis of the temperature pattern of the whole hands, avoiding the cold stress procedure. In fact, the experimental measure consisted of the acquisition of a single thermal image of both subject's hands in a resting condition.

A CNN was used to classify the images into two classes: healthy controls and SSc patients. The CNN adopted was able to classify the SSc subjects with a high degree of accuracy, around $82 \%$, very similar to the commonly long-lasting standard procedure. Notably, the choice of optimization algorithm for deep learning model is extremely important. In general, learning algorithms are procedures that optimize the network's biases and weights by exploring the parameters' 
space relying on the gradient of the objective function [55]. Hence, a future purpose is to validate the technique on a larger sample of subjects to further corroborate the generalization capabilities of the approach and to investigate the performances of the classification procedure accordingly to different choices of parameters.

Importantly, the possibility to classify SSc from healthy controls without the employment of a cold stimulus constitutes an advantage with respect to the standard procedure, particularly for clinical applications. In fact, the standard procedure, lasting around 25 minutes, does not allow for widespread clinical screening of the pathology, that could be fundamental for early diagnosis and treatment of the disease. However, it may be worth to investigate the capabilities of the machinery to classify SSc patients from people affected by other vascular diseases, in order to develop a robust and accurate procedure for the SSc diagnosis in clinical practice.

\section{Conclusion}

In this study, an innovative automatic SSc classification procedure based on CNN and IR images of the hands during a rest condition was presented. This approach allowed to overcome issues related to the administration of a cold stimulus to the patients, and to avoid biases in the classification introduced by the operator. Given the high accuracy (82.35\%) and the ecological features of the procedure (i.e. non-invasive, contactless, and short-lasting), it constitutes a promising tool for large scale SSc clinical screening and diagnosis, representing a great improvement with respect to the standard procedure. To the best of our knowledge, it is the first time that such an automatic approach is used for SSc classification.

\section{REFERENCES}

1. Nussinovitch, U. The heart in rheumatic, autoimmune and inflammatory diseases: pathophysiology, clinical aspects and therapeutic approaches; Academic Press, 2017; ISBN 0-12-803268-5.

2. Bernatsky, S.; Joseph, L.; Pineau, C.A.; Belisle, P.; Hudson, M.; Clarke, A.E. Scleroderma prevalence: Demographic variations in a population-based sample. Arthritis Care \& Research 2009, 61, 400-404.

3. Cutolo, M.; Sulli, A.; Smith, V. Assessing microvascular changes in systemic sclerosis diagnosis and management. Nature Reviews Rheumatology 2010, 6, 578.

4. Arias-Nunez, M.C.; Llorca, J.; Vazquez-Rodriguez, T.R.; Gomez-Acebo, I.; Miranda-Filloy, J.A.; Martin, J.; GonzalezJuanatey, C.; Gonzalez-Gay, M.A. Systemic sclerosis in northwestern Spain: a 19-year epidemiologic study. Medicine 2008, 87, 272-280.

5. Suarez-Almazor, M.E.; Kallen, M.A.; Roundtree, A.K.; Mayes, M. Disease and symptom burden in systemic sclerosis: a patient perspective. The Journal of Rheumatology 2007, 34, 1718-1726.

6. Gabrielli, A.; Avvedimento, E.V.; Krieg, T. Scleroderma. New England Journal of Medicine 2009, 360, $1989-2003$.

7. Abou-Raya, A.; Abou-Raya, S.; Helmii, M. Statins: potentially useful in therapy of systemic sclerosis-related Raynaud's phenomenon and digital ulcers. The Journal of Rheumatology 2008, 35, 1801-1808.

8. Mouthon, L.; Mestre-Stanislas, C.; Bérezné, A.; Rannou, F.; Guilpain, P.; Revel, M.; Pagnoux, C.; Guillevin, L.; Fermanian, J.; Poiraudeau, S. Impact of digital ulcers on disability and health-related quality of life in systemic sclerosis. Annals of the rheumatic diseases 2010, 69, 214-217.

9. El Sawy, N.; Suliman, I.; Nouh, M.; Naguib, A. Hand function in systemic sclerosis: A clinical and ultrasonographic study. The Egyptian Rheumatologist 2012, 34, 167-178.

10. Hudson, M.; Thombs, B.D.; Steele, R.; Panopalis, P.; Newton, E.; Baron, M.; Group, C.S.R. Health-related quality of life in systemic sclerosis: a systematic review. Arthritis Care \& Research 2009, 61, 1112-1120.

11. Ingegnoli, F.; Boracchi, P.; Ambrogi, F.; Meroni, P.L. Comparative analysis of different specific indices of hand impairment in systemic sclerosis. The Journal of rheumatology 2010, 37, 2192-2193.

12. Sapadin, A.N.; Fleischmajer, R. Treatment of scleroderma. Archives of dermatology 2002, 138, 99-105.

13. Nives Parodi, M.; Castagneto, C.; Filaci, G.; Murdaca, G.; Puppo, F.; Indiveri, F.; Scudeletti, M. Plicometer skin test: a new technique for the evaluation of cutaneous involvement in systemic sclerosis. British journal of rheumatology 1997, 36, 244-250.

14. Weibel, L.; Howell, K.J.; Visentin, M.T.; Rudiger, A.; Denton, C.P.; Zulian, F.; Woo, P.; Harper, J.I. Laser Doppler flowmetry for assessing localized scleroderma in children. Arthritis \& Rheumatism: Official Journal of the American College of Rheumatology 2007, 56, 3489-3495.

15. Garcia-Romero, M.T.; Randhawa, H.K.; Laxer, R.; Pope, E. The role of local temperature and other clinical characteristics of localized scleroderma as markers of disease activity. International journal of dermatology 2017, $56,63-67$.

16. Merla, A.; Di Donato, L.; Di Luzio, S.; Farina, G.; Pisarri, S.; Proietti, M.; Salsano, F.; Romani, G.L. Infrared functional imaging applied to Raynaud's phenomenon. IEEE Engineering in Medicine and Biology Magazine 2002, 21, 73-79.

17. Cardone, D.; Merla, A. New frontiers for applications of thermal infrared imaging devices: Computational psychopshysiology in the neurosciences. Sensors 2017, 17, 1042.

18. Filippini, C.; Spadolini, E.; Cardone, D.; Merla, A. Thermal Imaging Based Affective Computing for Educational Robot. Proceedings 2019, 27, 27, doi:10.3390/proceedings2019027027. 
19. Engert, V.; Merla, A.; Grant, J.A.; Cardone, D.; Tusche, A.; Singer, T. Exploring the Use of Thermal Infrared Imaging in Human Stress Research. PLoS One 2014, 9, doi:10.1371/journal.pone.0090782.

20. Filippini, C.; Perpetuini, D.; Cardone, D.; Chiarelli, A.M.; Merla, A. Thermal Infrared Imaging-Based Affective Computing and Its Application to Facilitate Human Robot Interaction: A Review. Applied Sciences 2020, 10, 2924.

21. Cardone, D.; Merla, A. The thermal dimension of psychophysiological and emotional responses revealed by thermal infrared imaging. In Proceedings of the 2014 IEEE International Conference on Image Processing (ICIP); IEEE, 2014; pp. 1942-1946.

22. de Campos, M.F.; Ripka, W.L.; Campos, D.; Heimbecher, C.T.; Esmanhoto, E.; Ulbricht, L. Raynaud's Phenomenon Differentiating After Cold Stress Using Thermal Parameters from Fingers. In Proceedings of the XXVI Brazilian Congress on Biomedical Engineering; Springer, 2019; pp. 869-874.

23. Danilova, V.A.; Shlykov, V.V. Use of thermal imaging for diagnosis of vascular pathologies. Біомедична інженерія та електроніка 2014.

24. Mariotti, A.; Grossi, G.; Amerio, P.; Orlando, G.; Mattei, P.A.; Tulli, A.; Romani, G.L.; Merla, A. Finger thermoregulatory model assessing functional impairment in Raynaud's phenomenon. Annals of biomedical engineering 2009, 37, 2631.

25. Ismail, E.; Orlando, G.; Corradini, M.L.; Amerio, P.; Romani, G.L.; Merla, A. Differential diagnosis of Raynaud's phenomenon based on modeling of finger thermoregulation. Physiological measurement 2014, 35, 703.

26. Sousa, E.; Vardasca, R.; Teixeira, S.; Seixas, A.; Mendes, J.; Costa-Ferreira, A. A review on the application of medical infrared thermal imaging in hands. Infrared Physics \& Technology 2017, 85, 315-323.

27. Krizhevsky, A.; Sutskever, I.; Hinton, G.E. Imagenet classification with deep convolutional neural networks. In Proceedings of the Advances in neural information processing systems; 2012; pp. 1097-1105.

28. Liang, M.; Hu, X. Recurrent Convolutional Neural Network for Object Recognition.; 2015; pp. 3367-3375.

29. LeCun, Y.; Bengio, Y.; Hinton, G. Deep learning. nature 2015, 521, 436-444.

30. Fu, H.; Niu, Z.; Zhang, C.; Ma, J.; Chen, J. Visual cortex inspired CNN model for feature construction in text analysis. Frontiers in computational neuroscience 2016, 10, 64.

31. Suh, S.C.; Anthony, T. Big Data and Visual Analytics; Springer, 2017; ISBN 3-319-63917-X.

32. Choudhury, B.; Then, P.H.H.; Raman, V. Automated Detection of Central Retinal Vein Occlusion Using Convolutional Neural Network. In Proceedings of the Big Data and Visual Analytics; Suh, S.C., Anthony, T., Eds.; Springer International Publishing: Cham, 2017; pp. 1-21.

33. Bhandare, A.; Bhide, M.; Gokhale, P.; Chandavarkar, R. Applications of convolutional neural networks. International Journal of Computer Science and Information Technologies 2016, 7, 2206-2215.

34. Kusumoto, D.; Yuasa, S. The application of convolutional neural network to stem cell biology. Inflammation and regeneration 2019, 39, 14.

35. Chiarelli, A.M.; Bianco, F.; Perpetuini, D.; Bucciarelli, V.; Filippini, C.; Cardone, D.; Zappasodi, F.; Gallina, S.; Merla, A. Data-driven assessment of cardiovascular ageing through multisite photoplethysmography and electrocardiography. Medical Engineering \& Physics 2019, 73, 39-50, doi:10.1016/j.medengphy.2019.07.009.

36. Ronneberger, O.; Fischer, P.; Brox, T. U-net: Convolutional networks for biomedical image segmentation. In Proceedings of the International Conference on Medical image computing and computer-assisted intervention; Springer, 2015; pp. 234-241.

37. Liu, W.; Huang, Q.; Chang, S.; Wang, H.; He, J. Multiple-feature-branch convolutional neural network for myocardial infarction diagnosis using electrocardiogram. Biomedical Signal Processing and Control 2018, 45, $22-32$.

38. Helsinki, D. of Recommendations guiding physicians in biomedical research involving human subjects. JAMA 1997, 277, 925-926.

39. Panasiti, M.S.; Cardone, D.; Pavone, E.F.; Mancini, A.; Merla, A.; Aglioti, S.M. Thermal signatures of voluntary deception in ecological conditions. Scientific reports 2016, 6, 1-10.

40. Thermology, I.A. of C. Thermography guidelines: standards and protocols in clinical thermographic imaging; IACT, 2002;

41. Bernard, V.; Staffa, E.; Mornstein, V.; Bourek, A. Infrared camera assessment of skin surface temperature-effect of emissivity. Physica Medica 2013, 29, 583-591.

42. Mitchell, D.; Wyndham, C.H.; Hodgson, T. Emissivity and transmittance of excised human skin in its thermal emission wave band. Journal of applied physiology 1967, 23, 390-394.

43. Mitchell, D.; Wyndham, C.H.; Hodgson, T.; Nabarro, F.R.N. Measurement of the total normal emissivity of skin without the need for measuring skin temperature. Physics in Medicine \& Biology 1967, 12, 359.

44. Hardy, J.D.; Muschenheim, C. The radiation of heat from the human body. IV. The emission, reflection, and transmission of infra-red radiation by the human skin. The Journal of clinical investigation 1934, 13, 817-831.

45. Derksen, W.L.; Monahan, T.I.; Lawes, A.J. Automatic recording reflectometer for measuring diffuse reflectance in the visible and infrared regions. JOSA 1957, 47, 995-999.

46. O'Shea, K.; Nash, R. An introduction to convolutional neural networks. arXiv preprint arXiv:1511.08458 2015

47. Dahl, G.E.; Sainath, T.N.; Hinton, G.E. Improving deep neural networks for LVCSR using rectified linear units and dropout. In Proceedings of the 2013 IEEE International Conference on Acoustics, Speech and Signal Processing; IEEE: Vancouver, BC, Canada, 2013; pp. 8609-8613.

48. Du, Y.; Fu, Y.; Wang, L. Skeleton based action recognition with convolutional neural network. In Proceedings of the 2015 3rd IAPR Asian Conference on Pattern Recognition (ACPR); IEEE, 2015; pp. 579-583. 
49. Bouchard, G. Clustering and classification employing softmax function including efficient bounds; Google Patents, 2011;

50. Sutskever, I.; Martens, J.; Dahl, G.; Hinton, G. On the importance of initialization and momentum in deep learning. In Proceedings of the International conference on machine learning; 2013; pp. 1139-1147.

51. Hastie, T.; Tibshirani, R.; Friedman, J. Overview of supervised learning. In The elements of statistical learning; Springer, 2009; pp. 9-41.

52. Kingma, D.P.; Ba, J. Adam: A method for stochastic optimization. arXiv preprint arXiv:1412.6980 2014.

53. Kohavi, R. A study of cross-validation and bootstrap for accuracy estimation and model selection. In Proceedings of the ljcai; Montreal, Canada, 1995; Vol. 14, pp. 1137-1145.

54. Abadi, M.; Barham, P.; Chen, J.; Chen, Z.; Davis, A.; Dean, J.; Devin, M.; Ghemawat, S.; Irving, G.; Isard, M. Tensorflow: A system for large-scale machine learning. In Proceedings of the 12th \{USENIX\} Symposium on Operating Systems Design and Implementation (\{OSDI\} 16); 2016; pp. 265-283.

55. Chiarelli, A.M.; Croce, P.; Merla, A.; Zappasodi, F. Deep learning for hybrid EEG-fNIRS brain-computer interface: application to motor imagery classification. Journal of neural engineering 2018, 15, 036028. 